\title{
PROPERTIES OF A MULTIDIMENSIONAL LANDSCAPE MODEL FOR DETERMINING CELLULAR NETWORK THERMODYNAMICS
}

Joseph B. Hubbard*, Michael Halter*, Anne L. Plant ${ }^{*} \&$

${ }^{*}$ National Institute of Standards and Technology, 100 Bureau Drive, Gaithersburg MD, 20899

\&Corresponding author: Anne Plant, 100 Bureau Drive, Gaithersburg MD, 20899, 301975 3124, anne.plant@nist.gov

\begin{abstract}
The steady state distributions of phenotypic responses within an isogenic population of cells result from both deterministic and stochastic characteristics of biochemical networks. A biochemical network can be characterized by a multidimensional potential landscape based on the distribution of responses and a diffusion matrix of the correlated dynamic fluctuations between $\mathrm{N}$-numbers of intracellular network variables. The Boltzmann $\mathrm{H}$-function defines the rate of free energy dissipation of a network system and provides a framework for determining the heat associated with the nonequilibrium steady state and its network components. We conjecture that there is an upper limit to the rate of dissipative heat produced by a biological system, and we show that the dissipative heat has a lower bound. The magnitudes of the landscape gradients and the dynamic correlated fluctuations of network variables are experimentally accessible, and through an analysis that we refer to as Thermo-Fokker-Planck (ThermoFP), provide insight into the composition of the network and the relative thermodynamic contributions from network components. We surmise that these thermodynamic quantities allow determination of the relative importance of network components to overall network control.
\end{abstract}

\section{INTRODUCTION}

Measurements of individual cells within a population indicate that phenotypic differences between isogenic cells is common, even when they are in a homogeneous and stable environment. Despite differences between individuals, populations of cells can manifest apparently stable distributions of phenotypic expression. The measured phenotypic parameters can include concentration of protein products of gene expression, indicators associated with promoter activation, RNA transcripts, and complex cell behavior such as morphology. The steady state distribution of phenotypes observed from 
single cell analysis is a probability density function and can be represented as a potential energy landscape (1-5).

Studies that involve imaging of live cells reveal that despite the observed consistency of the steady state, or stationary, distribution of phenotypes across the population of cells, there can significant dynamic variability in each cell and from one cell to another $(3,6-11)$. A number of studies provide direct evidence that the distribution of phenotypes in steady state distributions is ergodic in that subsequent to a transient perturbation the population will eventually relax to the steady state distribution $(3,12-16)$. The recapitulation of the steady state distribution, even after single cell cloning demonstrates that each cell or its progeny can explore all microstates on the landscape. The population response appears deterministic, but the individual entities (cells) that comprise the population present a dynamic, random expression of phenotypes, which ultimately results in the heterogeneity of the population. These are characteristics of many physical systems that can be well-described by statistical mechanics. The variations in populations of cells is often attributed to stochastic fluctuations, or noise, due to small numbers of molecules associated with transcription and translation. However, fluctuations in small numbers of molecules is not the only source of this variation $(8,17-19)$; correlation analysis has suggested that the main source of noise may be upstream regulatory components (12). Ensembles of biochemicals are responsible for producing an observed phenotype $(8,19,20)$, and these network components demonstrate coordinated concentration response functions. Importantly, dynamic fluctuations of network components, and correlations in fluctuations among multiple network components, are defining features of regulated networks $(2,14,19,21-27)$. An abbreviated biochemical network system is depicted in the schematic in Figure 1, based on the interactions between transcription factors involved in maintaining pluripotency (28), and provides an illustration of some of the general network features the our model addresses. 


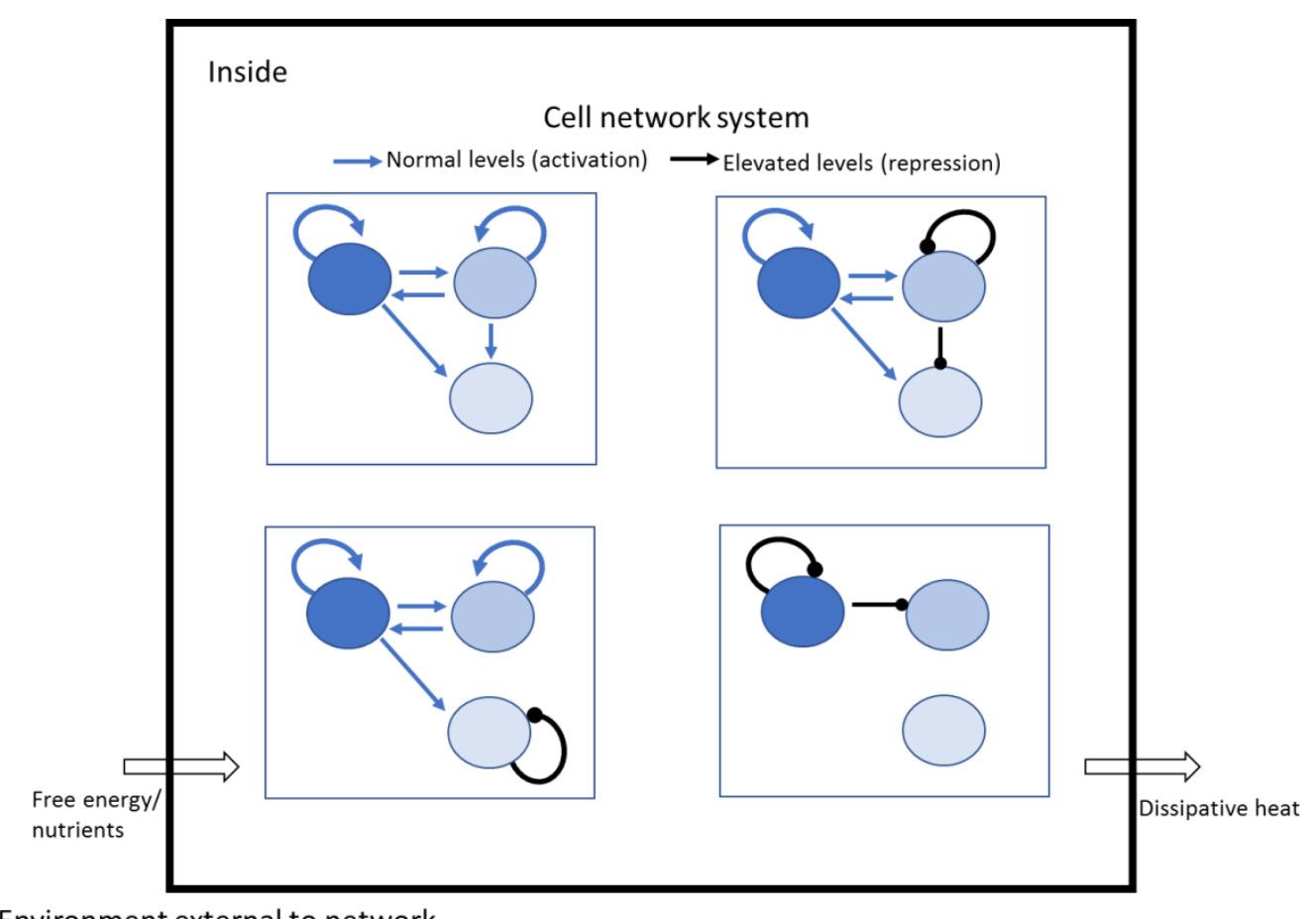

Environment external to network

Figure 1. Schematic of an intracellular network (based on a schematic by Rizzino and Wuebben 2016 (28) on control of pluripotency), consisting of $\mathrm{N}=3$ variable components, or dimensions, of a network with activation and repression relationships as indicated. As described in the text, these relationships define the measurable covariances in an NxN diffusion matrix, which contribute to the thermodynamics of the network together with the gradients of a landscape (which are derived from the multi-dimensional steady state probability density). The nonequilibrium steady state in this open thermodynamic system is supported by an influx of free energy from outside the system, which is dissipated as heat.

Identification of network components and the nature of their interdependence remains a challenge. While "omics" analysis methods can probe many variables simultaneously and even at the level of individual cells, they provide only a snapshot in time. While these methods allow determination of the coincidence of molecular species, without dynamic data in individual cells that can reveal the rates and magnitudes of the interactions between network species, it is impossible to determine unambiguously the relationship between them or to determine a physical mechanistic basis for control of the network $(10,19,29,30)$. A promising approach for observing single cell dynamics is imaging of live cells over time, which provides access to fluctuations in phenotypic expression in individual cells across a population $(9-11,14,24)$. Live cell imaging can also provide spatial and temporal information across scales and can provide ancillary information such as direct observation of the timing of cell division. We have previously used quantitative microscopy $(3,7)$ to track temporal responses of populations of 
individual cells, and Langevin/Fokker-Planck (L/FP) dynamics to analyze the population distributions as potential energy landscapes (3). By measuring real time trajectories of the activity of the gene promoter for the extracellular matrix protein tenascin $C$, we determined the diffusion coefficient for fluctuations in the promoter, and employing L/FP dynamics, accurately predicted the time dependent relaxation of subpopulations to the steady state distribution with no adjustable parameters (3). Such a onedimensional landscape is a simple manifestation of a complex adaptive system but provides little insight into the mechanisms responsible for establishing and maintaining the stability and dynamics of the steady state distribution because of the contributions from unseen variables. In the current theoretical work, we consider a network of $\mathrm{N}$ numbers of variables. An $\mathrm{N}$-dimensional statistical thermodynamics analysis allows fundamental questions about the thermodynamic controls in a coordinated network system to be addressed, including: how to identify the most important components of a regulated network, what is the relative thermodynamic contribution of different network components, and what is the thermodynamic price of homeostasis of a regulated network?

We present an experimentally accessible theoretical Fokker-Planck (FP) framework, which we refer to as Thermo-FP, that demonstrates that the rate of free energy dissipation associated with maintaining a nonequilibrium steady state network of intracellular reactions can be determined by the covariances in the temporal fluctuations in the components of the network and the gradients of the potential landscape. This analysis is enabled by recent application of FP dynamics (31) to demonstrate that the Boltzmann $\mathrm{H}$-function explicitly connects the relative entropy of a relaxing population with the rate of dissipation of free energy involved in maintaining the network at steady state.

\section{RESULTS}

\section{The theoretical framework}

The Fokker-Planck (FP) equation (32) describes the time evolution of the probability density of a population as it approaches a steady state distribution following a transient perturbation. This evolution is considered at small time intervals as a function of a deterministic component in the form of a drift vector, and a diffusion component that describes the stochastic fluctuations within the distribution. We present Thermo-FP dynamics to describe the evolution to a steady state of an $\mathrm{N}$-dimensional probability 
distribution with $\mathrm{N} \times \mathrm{N}$ diffusion matrices of variances and covariances in and between network variables. The magnitude of the coupled fluctuations between the different network variables, i.e., the covariances that comprise the diffusion matrix, is a measure of the strength of the physical and functional interaction between those variables (19). The steady state is a relatively low free energy, high entropy state. The fluctuations that occur around this state are states of higher free energy and lower entropy, and the magnitude of the measurable correlated fluctuations between network variables, which comprise the diffusion matrix, is related to heat through the Boltzmann constant (33). The coordinated relationships between network components create an organized structure, thus reducing entropy and resulting in the production of dissipative heat (34).

Here we treat multidimensional landscapes of biochemical networks by applying the mathematical properties of positive definite quadratic forms and normal mode analysis common to mathematical physics and statistical mechanics (35). A diffusion matrix of the dynamic covariances between the multiple variables of the network can be rotated through normal mode analysis to identify complex collective modes of network variables. The product of an eigenvalues of the rotated diffusion matrix with the square of the gradient of the rotated multidimensional landscape allows determination of the contribution each degree of freedom makes to the rate of dissipation of heat that maintains the regulated network of reactants.

We begin by showing that the Boltzmann $\mathrm{H}$ function describes the free energy of a population relaxing by Thermo-FP dynamics to a steady state distribution.

\section{The steady state landscape and the Boltzmann H Function}

The Boltzmann equation relates entropy, $S$, to the number of microstates in a distribution, $W$, of an equilibrated system: $S=k_{B} \ln W$. For a nonequilibrium steady state system, the occupation of microstates results from irreversible processes and the steady state is supported by influx of external free energy. For a multi-dimensional system of $\mathrm{N}$ network variables that is approaching its nonequilibrium steady state, entropy decreases over time: $S=-\int W(\{x\}, t) \ln W(\{x\}, t) d^{N} x$ where $W(\{x\}, t)$ is the occupation probability density of phenotypic microstates as a function of time and location on the $\mathrm{N}$-dimensional landscape. For both equilibrium and nonequilibrium systems, a minimum free energy is achieved when a system has relaxed to its stable steady state. During relaxation, which is dependent on stochastic fluctuations, relative free energy is dissipated as heat. The free energy of a 
system approaching equilibrium can be identified with the Boltzmann $\mathrm{H}$-function, $H(t)$, and $-d H / d t$ is the rate of dissipation of free energy as a system that is transiently perturbed away from its steady state relaxes to a steady state distribution, $W_{s S}$. In the following presentation, the factor $k T$ is assumed but not explicitly included in equations.

$H(t)$ is a relative free energy defined by

$$
H(t, \tau)=\int d^{N} x W_{1}(\{x\}, t) \ln \frac{W_{1}(\{x\}, t)}{W_{2}(\{x\}, t+\tau)}
$$

in terms of probability densities, where $W_{1}$ is the probability density of the microstates at some time, $t$ during relaxation and $W_{2}$ is the probability density at time $t+\tau$ (depicted schematically in Figure 2 ). Thus, the relative free energy of the system is defined by the average of the logarithm of the ratio of the occupation probabilities of the microstates, $\{x\}$, of the distribution. Keeping in mind that timedependent relaxation is determined by stochastic fluctuations of characteristics rates, a series of probability density functions is observed over time (Figure 1), each of which can be described by Thermo-FP dynamics in terms of a potential force, or drift, term and a diffusive term at every microstate; the former corresponds to the gradient of the landscape on which the microstate resides, and the latter is described in an $\mathrm{N}$ by $\mathrm{N}$ diffusion matrix of variances and covariances of the fluctuations of the $\mathrm{N}$ network variables (see SI 1). As the perturbed system relaxes, $H(t)$ decreases with time $\left(\frac{d H(t)}{d t} \leq 0\right)$ and is a minimum at steady state, $S S$.

The time derivative of the Boltzmann $\mathrm{H}$-function, $\frac{d H(t)}{d t}$ can be written as a quadratic form $(31,32)$, consistent with FP dynamics.

$$
\frac{d H(t)}{d t}=-\frac{1}{2} \sum_{i, j}^{N} \int d^{N} x W(\{x\}, t) \frac{\partial}{\partial x_{i}} \ln R \cdot D_{i j}(\{x\}) \cdot \frac{\partial}{\partial x_{j}} \ln R
$$

Here a measurable multi-dimensional probability density provides the relative free energy associated with the microstates of the landscape, $R=\frac{W(\{x\}, t)}{W_{S S}(\{x\})}$. The gradients of the landscape, $\frac{\partial}{\partial x_{i}} \ln R$ and 
$\frac{\partial}{\partial x_{j}} \ln R$ are analogous to the gradients of a chemical potential; and $D_{i j}$ is a diffusion matrix of experimentally determined covariances between the $\mathrm{N}$ variables $(36,37)$. The $\mathrm{N}$-dimensional diffusion matrix, $D_{i j}$, reflects the fluctuations in each of $\mathrm{N}$ network components, which are the diagonal elements (variances), and the dynamic correlations (covariances) between the $\mathrm{N}$ variables, which are the offdiagonal elements.

A

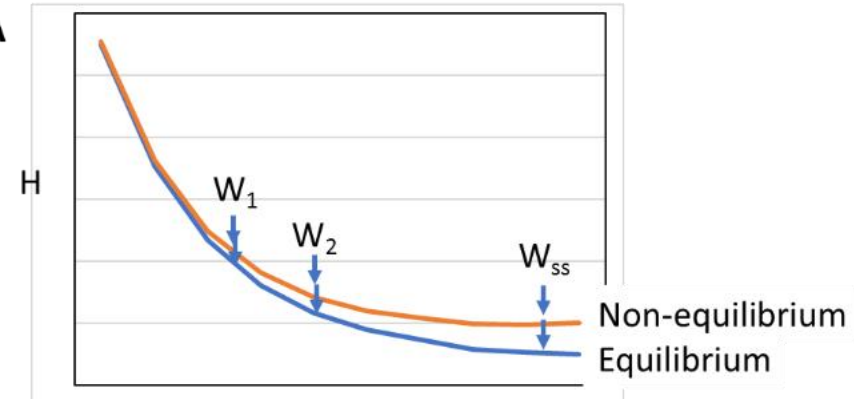

Time of Relaxation
B

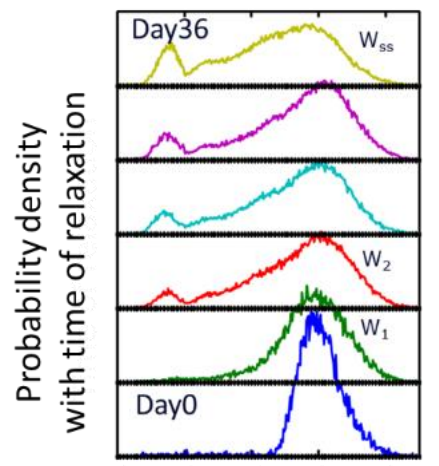

Figure 2. A steady state distribution, if transiently perturbed, will relax back to the steady state distribution. (Left) The rate of dissipation of free energy during relaxation, $\frac{d H(t)}{d t}$, approaches 0 as the steady state $\left(\mathrm{W}_{\mathrm{ss}}\right)$ is reached. A thermodynamically open, nonequilibrium system is less entropic, and of higher free energy, than an equilibrium system. (Right) $H(t)$ corresponds to population distributions (shown here for 1-dimensional distributions) (from Sisan et. al. 2012)(3). A subpopulation resulting from cell sorting (Day 0 ) is allowed to relax, and over time, the population achieves the initial steady state distribution from which the subpopulation was taken.

\section{The energetic components of the non-equilibrium steady state}

By setting $R(\{x\}, t)=\frac{W(\{x\}, t)}{W_{s S}(\{x\})}$ in Eq. 2, where $W_{s S}(\{x\})$ refers to a time-invariant steady state, we see that Thermo-FP dynamics ensures that $\frac{d H(t)}{d t}$ vanishes as the stationary state is approached and $R$ approaches unity and $\ln R(\{x\}, t)$ approaches 0 .

At equilibrium, the rate of increase in entropy and the rate of dissipation of free energy will approach zero. Living biological systems do not exist at equilibrium, and instead reach a nonequilibrium steady state with reduced entropy and higher free energy compared to an equilibrium state. The equilibrium 
state is defined by detailed balance, in that the probability flux between any pair of points on the landscape is equal to the probability flux in the reverse direction; in the nonequilibrium state there is a lack of reversibility. Interactions between network components create a coordinated set of reactions; this allows the system to respond dynamically to arbitrary perturbations in order to recover homeostasis. This molecular organization reduces entropy and keeps the system in a nonequilibrium steady state that is maintained by the injection of free energy from outside the system; that energy is dissipated by the system as heat (34). During relaxation to an equilibrium steady state, $\frac{d H(t)}{d t}$ is the rate of free energy dissipated from the production of entropy during approach to the steady state, i.e., $\mathrm{dH} / d t=-\dot{Q}_{E N T R O P Y}$. During relaxation to a nonequilibrium steady state distribution, $\frac{d H(t)}{d t}$ is the rate of free energy dissipated from two sources, namely $\dot{Q}_{E N T R O P Y}$, and also the rate of dissipation of heat from the free energy that is required to maintain the nonequilibrium steady state, $\dot{Q}_{E N E R G Y}$. (The dot indicates the time rate of change of the quantity). Thus, for a nonequilibrum system:

$$
\begin{gathered}
\dot{Q}_{E N E R G Y}(t) \geq 0, \dot{Q}_{\text {ENTROPY }}(t) \geq 0 \\
\frac{d H(t)}{d t}=\dot{Q}_{E N E R G Y}(t)-\dot{Q}_{E N T R O P Y}(t) \leq 0
\end{gathered}
$$

Before reaching steady state:

$$
\frac{d H(t)}{d t}=\frac{1}{2} \sum_{i, j}^{N} \int d^{N} x W(\{x\}, t)\left[\frac{\partial}{\partial x_{i}} \ln W_{S S}(\{x\}) \cdot D_{i j}(\{x\}) \cdot \frac{\partial}{\partial x_{j}} \ln W_{S S}(\{x\})\right]-\dot{Q}_{E N T R O P Y}(t) \leq 0
$$

At steady state:

$$
\dot{Q}_{E N E R G Y}=\frac{1}{2} \sum_{i, j}^{N} \int d^{N} x W(\{x\})\left[\frac{\partial}{\partial x_{i}} \ln W_{s S}(\{x\}) \cdot D_{i j}(\{x\}) \cdot \frac{\partial}{\partial x_{j}} \ln W_{S S}(\{x\})\right] \geq 0
$$

The Boltzmann $\mathrm{H}$ function thus provides us with a measure of the rate of heat dissipation, which we will call $\dot{Q}_{E N E R G Y}$, associated with maintaining the nonequilibrium steady state that is analogous to the 
"housekeeping heat" introduced by Oono and Paniconi (38) and further developed by others $(39,40)$.

We define $\dot{Q}_{E N E R G Y}$ as distinct from housekeeping heat because it is the dissipative heat that remains associated with a nonequilibrium steady state after $\frac{d H(t)}{d t}$ has reached zero. It is a value that can be determined in the absence of consideration of an equilibrium distribution, which is not experimentally observable in a biological system. At steady state the rate of heat dissipation due to $\dot{Q}_{E N E R G Y}$ is equal to the rate of entropy production $(39,41)$.

\section{Normal mode representation of the diffusion matrix}

The nonequilibrium steady state distribution of a cell population can be thought of as composed of microstates, $\{x\}$, the different ways the population can achieve its continuum of phenotypic expressions. The phenotypic expressions of the population are determined by the dynamic interactions of the components of the network; these interactions are represented in the $\mathrm{N} \times \mathrm{N}$ diffusion matrix, $D_{i j}(\{x\})$, as the autocorrelations and the dynamic covariances of the fluctuations of the activities of the $\mathrm{N}$ network variables. The dynamic covariances are the mean square fluctuations about the average relative expression levels divided by time. The $\mathrm{H}$ function provides us with the time-derivative of the relative free energy of the system, from which we can identify $\dot{Q}_{E N E R G Y}(t)$, the rate at which heat is dissipated during the maintenance of the nonequilibrium steady state of the network. We will use these relationships to determine the relative rate of heat dissipation by the various network components to support the homeostatic steady state distribution.

The normal modes of the diffusion matrix result from rotation of the matrix $\left(D_{i j}(\{x\})\right)$ to a diagonal form and provide us with a matrix $\left(D_{i i}\left(\left\{x^{*}\right\}\right)\right)$ in which the eigenvalues of all diagonal elements are greater than 0 and the off-diagonal elements are equal to 0 , i.e., the components of the matrix are independent of one another. The purpose of this transformation is to identify combinations of network components as composite variables that are the major contributors to the rate of heat dissipation of the system; these are effectively the degrees of freedom. The original diffusion matrix represents the pair-wise interactions between network variables, some of which are positive and some negative. Rotation of the matrix allows us to define composite variables (degrees of freedom) as clusters of $\mathrm{N}$-wise interactions between the $\mathrm{N}$ components of the network. The rotation operation guarantees eigenvalues that are positive as well as eigenvectors that are mutually orthogonal. We transpose integration and summation (42) operations in Eq. 6 to yield an expression for the energetics of the system as 


$$
\dot{Q}_{E N E R G Y}(t)=\int d^{N} x^{*} W\left(\left\{x^{*}, t\right\}\right) \cdot \sum_{i}^{N} \lambda_{i}\left(\left\{x^{*}\right\}\right) V_{i}^{2}\left(\left\{x^{*}\right\}\right)
$$

In Eq.7, we isolate terms within the summation operator to better illustrate their energetic contributions to the network. In Eq. 7, $\lambda_{i}\left(\left\{x^{*}\right\}\right)$ are the eigenvalues of the rotated diffusion matrix and represent interactions between network components and the magnitude of their coordinated fluctuations. Eigenvectors represents ways, i.e., modes, in which network components are organized with respect to their interactions with one another. The eigenvalues are the diffusion coefficients associated with those independent collective modes of the network components. The term $V_{i}\left(\left\{x^{*}\right\}\right)$ is equal to the gradient of the potential defined by the rotated landscape and expressed as $\left[V_{i}^{2}\left(\left\{x^{*}\right\}\right)\right]=$

$\left[\frac{\partial}{\partial x_{i}{ }^{*}} \operatorname{lnW}_{s s}\left(\left\{x^{*}\right\}\right)\right]^{2}$. It should be noted that the eigenvalues as well as the gradients of the potentials are functions of the entire coordinate set of $x^{*}$. Eq. 7 shows that the rate of free energy dissipation to maintain the nonequilibrium steady state will be largest when eigenvalues are large because of strong dynamic interactions between components of the network and when those interactions are occurring in a part of the landscape that is characterized by steep gradients. The rotated diffusion matrix produces positive eigenvalues and corresponding orthogonal eigenvectors, which simplifies the next step which is to sum the most important contributors to the rate of free energy utilization and heat dissipation.

\section{Network contributions to $\dot{Q}_{E N E R G Y}$}

$\dot{Q}_{E N E R G Y}$ in Eq. 7 is the rate at which the N-dimensional network is dissipating heat associated with the maintenance of the nonequilibrium steady state. It can also be regarded as the rate at which external free energy is injected into the network. The summation term on the right side of Eq. 7 is a positive definite quadratic form; in keeping with statistical thermodynamics we use this term to determine the energy that each of the degrees of freedom contribute to the system. The magnitude of $\lambda_{i}\left(\left\{x^{*}\right\}\right)$ and $\left[V_{i}\left(\left\{x^{*}\right\}\right)\right]^{2}$ in $\dot{Q}_{E N E R G Y}$ integrated over the probability distribution defines the rate of heat dissipation by the various components of the homeostatic network. This treatment implies that some interactions between network components or variables are more dissipative than others and therefore are more important thermodynamic contributors to the steady state. Below we will address the significance of this. 
If we consider each composite network variable that is identified by rotation of the matrix as a contributor of a degree of freedom, their sum, $C$, represents the total rate of heat dissipation associated with the system as shown in Eq. 8.

$$
C=\sum_{i}^{N} \lambda_{i}\left[V_{i}^{*}\right]^{2}
$$

Each term is an implicit function of $x^{*}$ and reflects a heat that is dependent on the magnitudes of the eigenvalues of the matrix, $\lambda_{i}$, and the square of the gradient, $V_{i}^{*}$, of the landscape that corresponds to the rotated coordinate system. Together these terms constitute a quadratic representation of the microscopic dissipation of the regulated circuit, a sequence of partial sums that increases monotonically as the number of terms increases. We are proposing that reactions that involve high rates of free energy dissipation are more important to the function of the cell vis a vis stability and adaptability.

We now make an assumption that for a biological system, there will likely be an upper bound, $C_{U B}$ on the local rate of dissipative heat produced. This upper limit may arise from the temperature associated with heat generation or the limited rate of transport of energy or matter from the environment into the system. The value of the sum of the energetics of the network components thus cannot exceed the upper bound denoted by:

$$
C_{U B} \geq C=\lambda_{1} V_{1}^{* 2}+\lambda_{2} V_{2}^{* 2}+\lambda_{3} V_{3}^{* 2}+\cdots \lambda_{N} V_{N}^{* 2}
$$

Each of the elements in Eq. 9 is a contributor to the summation of network dissipative heat. This treatment thus provides us, in principle, with an experimentally tractable way of assessing the relative contribution that each multivariable component makes to the rate of heat dissipation in the maintenance of the network, and the free energy cost associated with keeping the network in homeostasis. When the number of network dimensions, $\mathrm{N}$, is sufficiently large, $C$ reaches a limit, $C_{U B}$ and after integrating over the multidimensional probability distribution this constant rate is identical to 
$\dot{Q}_{\text {ENERGY }}$. A geometric approach for estimating the size of $\mathrm{N}$ required for convergence of $C$ to $C_{U B}$ is described in SI 3.

In addition to the assumption of an upper bound on dissipative heat, we utilize a generalization of the maximum entropy principle to show that the homeostatic heat generation rate, $\dot{Q}_{E N E R G Y}$, also has a lower bound, i.e., there exists a finite dissipative gap between the non-equilibrium stationary state and the equilibrium (detailed balance) distribution (see SI 4).

\section{DISCUSSION}

While it has been frequently noted that stochastic fluctuations in molecular components in individual cells are important to regulatory mechanisms in one-dimensional systems $(12,24,43,44)$ and in multidimensional networks $(21,25,27,45,46)$, to our knowledge, this work is the first to show the relationship between fluctuations in network variables and the thermodynamic quantities associated with the interactions between network components. We have limited our theoretical Thermo-FP approach to one that is experimentally tractable. We have shown here how the use of a potential landscape and a diffusion matrix provides a framework for determining the relative energetic contributions of the components of a regulated network. We derive an experimentally accessible value for $\dot{Q}_{E N E R G Y}$, the rate of heat dissipation associated with maintaining the nonequilibrium steady state and the analog of "housekeeping heat", directly from the Boltzmann $\mathrm{H}$ function. While we have presented this method as applied to analyzing steady state distributions, this approach is also applicable to population state transitions as discussed in SI 1.

Large magnitudes of correlated fluctuations of network components are associated with large rates of heat dissipation (41), and our theoretical treatment shows that this is especially true when these fluctuations occur in areas of steep landscape gradients. Noise and complexity are defining features of biological systems, and the Thermo-FP analysis suggests a thermodynamic basis for the relationship between noise and complexity and the stability of a regulated network. Fluctuations are required to ensure ergodicity by allowing cells to escape from deep attractor basins and maintain the stability of the entire landscape structure. We may consider that a biological system requires both stability and adaptability even though these may seem to be opposing characteristics. The Thermo-FP treatment presented here provides a thermodynamic basis for understanding why both the diffusion matrix and barrier gradients are important for maintaining the distribution of phenotypes. Deeper attractor basins 
associated with non-negligible diffusion coefficients enable stability of the network landscape during nominally constant environmental conditions, and shallower attractors allow adaptation to changing conditions through transition to a new steady state. Very small diffusion coefficients could result in longlived metastable states, analogous to glassy conditions.

The reasonable assumption that there will be a limit upper to the rate of heat dissipation in a biological system suggests that characteristics of landscapes and diffusion coefficients provide insight into numbers of network variables, stability, and composition of the network. There exists a geometric estimation of the number, $\mathrm{N}$, of variables required for convergence in terms of the volumes of $\mathrm{N}$-balls and N-ellipsoids (47). Depending on characteristics of the system, such as roughness of the landscape, sufficient numbers of network terms can be predicted to be as small as a few, or many times larger. A reasonable estimate for the number of variables is between 8 and 10. How a sufficient number of terms for convergence to $\mathrm{N}$ can be determined is discussed in SI 3. For example, very large landscape gradients that correspond to large eignenvalues would contribute strongly to the overall dissipative heat of the network, and a small number of such contributors may be sufficient to reach a thermal limit. This condition would be indicated by a landscape containing one or a small number of very deep attractor basins.

The measurement of dynamic correlations of many cellular variables over time in individual cells is in principle achievable with time-resolved fluorescence microscopy of live cells (see SI 2 for details), especially when enabled by automation and advances in handling of large image datasets $(48,49)$. Although transcriptomics analysis can probe a larger number of genes compared to live cell imaging, the appropriate interpretation of the relative significance of these changes to network function and their relationships to one another can be ambiguous $(19,29)$. Methods like transcriptomics analysis that rely on "snapshots" of populations at single points in time can infer temporal and treatment-dependent relationships between variables, but real time trajectories of changes in gene expression in individual cells, such as is accessible by live cell imaging, can provide unambiguous determination of correlations in stochastic fluctuations between network variables. In the absence of such direct measurements, a number of approaches have been pursued for inferring individual cell trajectories from population data including population balance analysis (29), optimum transport (50), and others discussed in (50). For these methods to be analytically tractable, a simplifying assumption is made that the stochastic component is Gaussian and uniform over gene expression space (as discussed further in SI 1). 
In Thermo-FP, stochasticity is captured in in the dynamic fluctuations of the network variables. The magnitudes of the correlations in dynamic fluctuations provide a direct measure of the thermodynamics of network component interactions (since the magnitudes of the fluctuations are proportional to $k_{B} T$, Boltzmann's constant times temperature), and thus the relative energetic significance of their contribution to the network. The correlations in fluctuations between each pair of $\mathrm{N}$-variables of the network are assigned to an $\mathrm{N} \times \mathrm{N}$ diffusion matrix for each microstate. The rate of heat dissipation associated with each composite variable is determined by the magnitude of the fluctuations and the gradient of the landscape. A variable that is a negligible contributor to dissipative heat would be predicted to play a small role in maintaining the network, and could indicate that the variable is coincidental, but not causative, to network function. A possible alternative method to assign relative importance to network components is surprisal analysis, which uses the maximum entropy associated with the time-dependent distribution of variables (51). To achieve unambiguous interpretation, we show here how these putative relationships can be assessed experimentally by directly measuring trajectories in variable space and their covariance.

This analysis has potential practical implications. $\dot{Q}_{E N E R G Y}$ is a quantitative measure of the rate of heat produced to maintain a regulatory network. The magnitude of $\dot{Q}_{E N E R G Y}$ can be used to compare the relative thermodynamic cost of different steady-state phenotypic distributions. For example, as a metric it could provide insight into the thermodynamics of different regulatory networks, or the same network functioning in cells from different individuals. It will be a useful metric to guide cell therapy manufacturing conditions, and to guide the engineering of regulatory pathways in synthetic biology applications.

\section{CONCLUSIONS}

Thermo-FP analysis, through rigorous connection to the Boltzmann $\mathrm{H}$ function and the rate of dissipation of free energy of the nonequilibrium steady state, provides a direct relationship between composite network variables and their contribution to the heat of maintaining homeostasis of the network.

The application of the Thermo-FP approach allows dynamical analysis of network interactions and cell states on a smooth continuous landscape. A multidimensional (or multi-variable) landscape that 
considers the dynamics of network components can provide unique understanding of the correct interpretation of cellular phenotypic indicators in the context of other network components, stable attractor states and rates at which neighboring phenotypic states can be accessed. Furthermore, we have shown how $\dot{Q}_{E N E R G Y}$, the dissipative heat required to maintain a multi-variable network can, in principle, be determined from experimentally tractable data consisting of a steady state distribution and a diffusion matrix of dynamic covariances in network variables. Each eigenvalue/eigenvector of the Ndimensional rotated matrix represents a unique and independent cluster of cooperative interactions of network components, and each constitutes a degree of freedom of the network.

This approach to analysis of the landscape provides unique insight into the components, paths, and the thermodynamic price associated with maintaining a nonequilibrium stationary multidimensional landscape of a regulated network. While we have focused this analysis on the steady state distribution, this approach will also be useful for tracking how cellular populations transition from one steady state to another in response to environmental changes, by helping to identify and quantify interactions between network components during transitions.

ACKNOWLEDGEMENTS. This work was funded with Scientific and Technical Research and Services funds by the National Institute of Standards and Technology

\section{REFERENCESReference List}

1. Huang S (2009) Reprogramming cell fates: reconciling rarity with robustness. Bioessays 31(5):546-560.

2. Garcia-Ojalvo J \& Martinez Arias A (2012) Towards a statistical mechanics of cell fate decisions. Curr Opin Genet Dev 22(6):619-626.

3. Sisan DR, Halter M, Hubbard JB, \& Plant AL (2012) Predicting rates of cell state change caused by stochastic fluctuations using a data-driven landscape model. Proc Natl Acad Sci U S A 109(47):19262-19267.

4. Sasai M, Kawabata Y, Makishi K, Itoh K, \& Terada TP (2013) Time scales in epigenetic dynamics and phenotypic heterogeneity of embryonic stem cells. PLoS Comput Biol 9(12):e1003380.

5. Morris R, Sancho-Martinez I, Sharpee TO, \& Izpisua Belmonte JC (2014) Mathematical approaches to modeling development and reprogramming. Proc Natl Acad Sci U S A 111(14):5076-5082. 
6. Kaufmann BB, Yang Q, Mettetal JT, \& van Oudenaarden A (2007) Heritable stochastic switching revealed by single-cell genealogy. PLoS Biol 5(9):e239.

7. Halter $\mathrm{M}$, et al. (2011) Cell cycle dependent TN-C promoter activity determined by live cell imaging. Cytometry A 79A(3):192-202.

8. Battich N, Stoeger T, \& Pelkmans L (2015) Control of Transcript Variability in Single Mammalian Cells. Cell 163(7):1596-1610.

9. Singer ZS, et al. (2014) Dynamic heterogeneity and DNA methylation in embryonic stem cells. Mol Cell 55(2):319-331.

10. Filipczyk A, et al. (2015) Network plasticity of pluripotency transcription factors in embryonic stem cells. Nat Cell Biol 17(10):1235-1246.

11. Patsch K, et al. (2016) Single cell dynamic phenotyping. Sci Rep 6:34785.

12. Sigal A, et al. (2006) Variability and memory of protein levels in human cells. Nature 444(7119):643-646.

13. Chang $\mathrm{HH}$, Hemberg M, Barahona M, Ingber DE, \& Huang S (2008) Transcriptome-wide noise controls lineage choice in mammalian progenitor cells. Nature 453(7194):544-547.

14. Kalmar T, et al. (2009) Regulated fluctuations in nanog expression mediate cell fate decisions in embryonic stem cells. PLoS Biol 7(7):e1000149.

15. Gupta PB, et al. (2011) Stochastic state transitions give rise to phenotypic equilibrium in populations of cancer cells. Cell 146(4):633-644.

16. Jafarpour F (2019) Cell Size Regulation Induces Sustained Oscillations in the Population Growth Rate. Physical Review Letters 122(11):118101.

17. Newman JR, et al. (2006) Single-cell proteomic analysis of S. cerevisiae reveals the architecture of biological noise. Nature 441(7095):840-846.

18. Balazsi G, van Oudenaarden A, \& Collins JJ (2011) Cellular decision making and biological noise: from microbes to mammals. Cell 144(6):910-925.

19. Prill RJ, Vogel R, Cecchi GA, Altan-Bonnet G, \& Stolovitzky G (2015) Noise-driven causal inference in biomolecular networks. PLoS One 10(6):e0125777.

20. Kontogeorgaki S, Sanchez-Garcia RJ, Ewing RM, Zygalakis KC, \& MacArthur BD (2017) Noiseprocessing by signaling networks. Sci Rep 7(1):532.

21. Huang S (2012) The molecular and mathematical basis of Waddington's epigenetic landscape: a framework for post-Darwinian biology? Bioessays 34(2):149-157.

22. Eldar A \& Elowitz MB (2010) Functional roles for noise in genetic circuits. Nature 467(7312):167173.

23. Li C \& Balazsi G (2018) A landscape view on the interplay between EMT and cancer metastasis. NPJ Syst Biol Appl 4:34.

24. Dunlop MJ, Cox RS, 3rd, Levine JH, Murray RM, \& Elowitz MB (2008) Regulatory activity revealed by dynamic correlations in gene expression noise. Nat Genet 40(12):1493-1498.

25. Pakka VH, Prugel-Bennett A, \& Dasmahapatra S (2010) Correlated fluctuations carry signatures of gene regulatory network dynamics. J Theor Biol 266(3):343-357.

26. Cox CD, McCollum JM, Allen MS, Dar RD, \& Simpson ML (2008) Using noise to probe and characterize gene circuits. Proc Natl Acad Sci U S A 105(31):10809-10814.

27. Azhar F \& Bialek W (2010) When are correlations strong? arXiv 1012.5987v1.

28. Rizzino A \& Wuebben EL (2016) Sox2/Oct4: A delicately balanced partnership in pluripotent stem cells and embryogenesis. Biochim Biophys Acta 1859(6):780-791.

29. Weinreb C, Wolock S, Tusi BK, Socolovsky M, \& Klein AM (2018) Fundamental limits on dynamic inference from single-cell snapshots. Proc Natl Acad Sci U S A 115(10):E2467-E2476.

30. Tkačik G \& Bialek W (2009) Diffusion, dimensionality, and noise in transcriptional regulation. Physical Review E 79(5):051901. 
31. Hubbard JB, Lund SP, \& Halter M (2013) Boltzmann's H-Function and Diffusion Processes. J Phys Chem B 117(42):12836-12843.

32. Risken H (1984) Fokker-Planck Equation. The Fokker-Planck Equation: Methods of Solution and Applications, ed Risken H (Springer Berlin Heidelberg, Berlin, Heidelberg), pp 63-95.

33. Attard P (2012) Non-equilibrium Thermodynamics and Statistical Mechanics: Foundations and Applications (Oxford Scholarship Online).

34. Prigogine I (1978) Time, structure, and fluctuations. Science 201(4358):777-785.

35. Courant RH, D. (2004) Methods of Mathematical Physics (WILEY-VCH Verlag GmbH \& Co. KGaA).

36. Bergmann PG \& Lebowitz JL (1955) New Approach to Nonequilibrium Processes. Phys Rev 99(2):578-587.

37. Lavenda BH \& Santamato E (1981) The Kinetic Analog of Boltzmanns Principle. J Math Phys 22(12):2926-2933.

38. Oono Y \& Paniconi M (1998) Steady state thermodynamics. Prog Theor Phys Supp (130):29-44.

39. Ge H \& Qian H (2010) Physical origins of entropy production, free energy dissipation, and their mathematical representations. Phys Rev E 81(5).

40. Speck T \& Seifert U (2005) Integral fluctuation theorem for the housekeeping heat. J Phys aMath Gen 38(34):L581-L588.

41. Wang J, Xu L, \& Wang E (2008) Potential landscape and flux framework of nonequilibrium networks: robustness, dissipation, and coherence of biochemical oscillations. Proc Natl Acad Sci U S A 105(34):12271-12276.

42. Rudin W (1987) Real and complex analysis (McGraw-Hill, New York) 3rd Ed pp xiv, $416 \mathrm{p}$.

43. Raj A \& van Oudenaarden A (2009) Single-molecule approaches to stochastic gene expression. Annu Rev Biophys 38:255-270.

44. Austin DW, et al. (2006) Gene network shaping of inherent noise spectra. Nature 439(7076):608611.

45. Tomioka R, Kimura H, T JK, \& Aihara K (2004) Multivariate analysis of noise in genetic regulatory networks. J Theor Biol 229(4):501-521.

46. Pedraza JM \& van Oudenaarden A (2005) Noise propagation in gene networks. Science 307(5717):1965-1969.

47. Wilson JA (2010) Volume of n-dimensional ellipsoid. Sciencia Acta Xaveriana 1(1):101-106.

48. Bajcsy PC, J.; Simon, M. (2018) Web Analysis of Big Image Data (Springer International Publishing).

49. Bhadriraju K, et al. (2016) Large-scale time-lapse microscopy of Oct4 expression in human embryonic stem cell colonies. Stem Cell Res 17(1):122-129.

50. Schiebinger G, et al. (2019) Optimal-Transport Analysis of Single-Cell Gene Expression Identifies Developmental Trajectories in Reprogramming. Cell 176(6):1517.

51. Remacle F, Kravchenko-Balasha N, Levitzki A, \& Levine RD (2010) Information-theoretic analysis of phenotype changes in early stages of carcinogenesis. Proc Natl Acad Sci U S A 107(22):1032410329. 